\title{
Unchartered territory
}

\author{
The chirped pulse amplification scheme celebrated by this year's Nobel Prize in Physics is allowing scientists to \\ investigate light-matter interaction at extreme regions of intensity and time.
}

$\int$ cience is sometimes prone to hyperbole and exaggeration. However, it is not an overstatement to say that the field of ultra-intense laser science, the domain of lasers emitting pulses with terawatt and petawatt peak powers, would likely not exist without the techniques celebrated in one half of this year's Nobel Prize in Physics ${ }^{1}$. Today, such lasers play a pivotal role in the ongoing global efforts to realize laser-based particle accelerators, inertial confinement fusion, and explore fundamental high-field physics at extreme scales ${ }^{2}$.

There is no doubt that the chirped pulse amplification (CPA) scheme, developed by Gérard Mourou and Donna Strickland in the 1980s at the University of Rochester, USA, unlocked a new frontier for laser science. It, overnight, provided a much-needed upgrade strategy for scaling the energy of laser pulses, opening the door to rich new opportunities for both science and applications.

The CPA scheme is now routinely used in labs around the world whenever scientists wish to have access to short, intense pulses of light. "All our experiments are done with CPA lasers," commented Bjorn Hegelich, who is Associate Director of the Center for Relativistic Laser Science at Gwangju Institute of Science \& Technology (GIST) in Korea and also a professor at the University of Texas at Austin, USA, which hosts the Texas Petawatt Laser. "The impact of CPA on the field of laser physics can hardly be overstated. Time resolution on the femtosecond and even attosecond scale at intensities required for imaging or pump-probe set-ups would not be possible otherwise. CPA lasers are also now a main part to be found in supporting roles on all our modern accelerator facilities like XFEL or LCLS, driving the electron sources and providing probe capabilities."

CPA also played an important role in the development of the field of attosecond science, where short intense laser pulses are used to ionize an atom in a highly energetic manner, resulting in high-harmonic generation and the production of very shortwavelength pulses of light with a duration on the sub-femtosecond scale. These extremely short pulses have become a useful tool for probing the ultrafast dynamics of electrons inside atoms and molecules.

"CPA has enabled us to generate controlled electric-field waveforms in the mid-infrared, which are useful for imaging

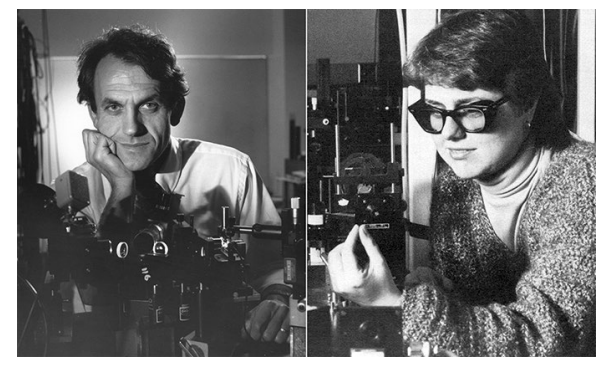

Credit: University of Rochester

chemical processes with atomic resolution via single-electron diffraction or to investigate the carrier dynamics in materials with attosecond X-ray pulses," commented Jens Biegert, head of the Attosecond and Ultrafast Optics group at ICFO in Barcelona, Spain.

In common with the early days of laser science, where development of the first lasers was largely inspired by research in another region of the electromagnetic spectrum (masers, which operate in the microwave region), CPA was inspired by work on radar and the amplification of radio-frequency $(\mathrm{RF})$ pulses. It was a solution to a pressing problem. The peak power and pulse energy of laser pulses had initially grown rapidly in the 1960s following the invention of the laser, but it soon hit a roadblock. Amplification of laser pulses to energies beyond the order of a millijoule proved problematic because the high light intensities involved induced the nonlinear effect of self-focusing and caused damage to the amplifier crystal and other optical components.

A similar situation arises in radar where the desire to obtain short, energetic RF pulses with high peak power can cause damage to electronic circuits. The radar community had solved the problem by stretching the RF pulses in time using a delay line with positive dispersion in order to reduce the peak power, then amplifying the longer pulse and finally compressing it once again under a negatively dispersive delay line.

The breakthrough innovation of Strickland and Mourou was to realize that the same stretch-amplify-compress technique could in principle be used in the optical domain to generate intense, ultrashort laser pulses.

In 1985, in what is now recognized as being a seminal paper in Optics Communications ${ }^{3}$, Strickland and Mourou outlined the CPA approach using a $1.4 \mathrm{~km}$ length of optical fibre as a stretcher and a pair of gratings as a compressor, and experimentally showed that they could generate $\sim 2$-ps-duration pulses with energies of $1 \mathrm{~mJ}$.

Today, these numbers may not sound very impressive but at the time it was an important demonstration that CPA worked. Furthermore, Strickland and Mourou had the foresight to see the potential scalability of the approach, writing in their paper: "Conceivably, this technique could be used to generate subpicosecond pulses with energies at the joule level." The rest, as they say, is history.

Currently, the scaling associated with the use of CPA and larger (metre-scale) optical components that are carefully engineered to have high damage-thresholds has taken laser pulse energies and peak powers to unprecedented values at numerous petawatt laser facilities around the world - such as Vulcan and Orion in the UK, GIST, Texas Petawatt Laser, OMEGA at Rochester, National Ignition Facility in LLNL, Laser Mégajoule in France, and FIREX at Osaka, Japan, to name just a few ${ }^{4}$.

"At GIST, we have what is currently the most powerful operating laser in the world at 4 PW," explained Hegelich. "The CPA pulse of this system can contain up to $80 \mathrm{~J}$ in $20 \mathrm{fs}$ on target and has been focused to an intensity of $6 \times 10^{22} \mathrm{~W} \mathrm{~cm}^{-2}$, using an $\mathrm{F} / 1.5$ off-axis parabola."

And there are plans to scale to even higher powers and intensities. The Extreme Light Infrastructure - Nuclear Physics (ELI-NP), a European project initiated by Mourou, which is now in the final stages before beginning operation in Romania, will house $10 \mathrm{PW}$ lasers, with the aim of reaching intensities of $10^{23} \mathrm{~W} \mathrm{~cm}^{-2}$ and electrical fields of $10^{15} \mathrm{~V}$ $\mathrm{m}^{-1}$. One hundred PW systems are planned for Shanghai (SEL) and Rochester (OPAL), for example. "To reach significantly higher powers than today, to go to exawatts or beyond, might very well require plasma optics to get around the damage thresholds," commented Hegelich. "However, as plasmas are very hard to control these will represent a tremendous challenge."

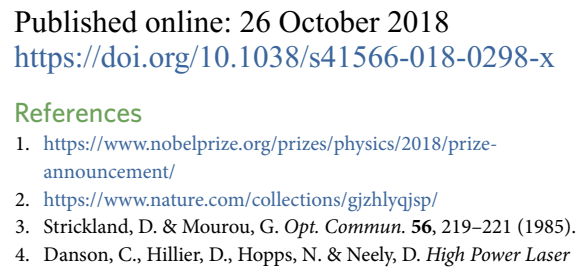

